\title{
A PET/CT volumetric parameter predicts prognosis of non-small cell lung cancer treated using preoperative chemoradiotherapy and surgery: A retrospective case series study
}

\author{
KUNIAKI KATSUI ${ }^{1}$, TAKESHI OGATA ${ }^{2}$, AKIHIRO TADA ${ }^{3}$, KENTA WATANABE ${ }^{4}$, \\ KOTARO YOSHIO ${ }^{4}$, MASAHIRO KURODA $^{5}$, KATSUYUKI KIURA $^{6}$, TAKAO HIRAKI $^{7}$, \\ SHINICHI TOYOOKA ${ }^{8}$ and SUSUMU KANAZAWA ${ }^{7}$
}

\begin{abstract}
${ }^{1}$ Department of Proton Beam Therapy, Okayama University Graduate School of Medicine, Dentistry and Pharmaceutical Sciences, Okayama 700-8558; ${ }^{2}$ Department of Radiology, Iwakuni Clinical Center, Iwakuni, Yamaguchi 740-8510; ${ }^{3}$ Department of Radiology, Okayama Diagnostic Imaging Center, Okayama 700-0913;

${ }^{4}$ Department of Radiology, Okayama University Hospital; ${ }^{5}$ Department of Radiological Technology, Graduate School of Health Sciences, Okayama University; ${ }^{6}$ Department of Allergy and Respiratory Medicine, Okayama University Hospital; Departments of ${ }^{7}$ Radiology and ${ }^{8}$ General Thoracic Surgery and Breast and Endocrinological Surgery, Okayama University Graduate School of Medicine, Dentistry and Pharmaceutical Sciences, Okayama 700-8558, Japan
\end{abstract}

Received September 18, 2020; Accepted December 17, 2020

DOI: $10.3892 / \mathrm{mco} .2021 .2235$

\begin{abstract}
The purpose of the present study was to clarify whether positron emission tomography/computed tomography (PET/CT) volumetric parameters were prognostic predictors of non-small cell lung cancer (NSCLC) treatment in patients who had undergone preoperative concurrent chemoradiotherapy (CCRT) and surgery. In the present study, retrospectively surveyed the data of patients with NSCLC who underwent preoperative CCRT and surgery at Okayama University Hospital (Okayama, Japan) between April 2006 and March 2018. The maximum standardized uptake value $\left(\mathrm{SUV}_{\max }\right)$ and volumetric parameters, including metabolic tumor volume (MTV) and total lesion glycolysis (TLG), were calculated using PET/CT and the percentage decrease $(\Delta)$ in
\end{abstract}

Correspondence to: Dr Kuniaki Katsui, Department of Proton Beam Therapy, Okayama University Graduate School of Medicine, Dentistry and Pharmaceutical Sciences, 2-5-1 Shikata-cho, Kita-ku, Okayama 700-8558, Japan

E-mail: kukatsui@cc.okayama-u.ac.jp

Abbreviations: $\quad{ }^{18} \mathrm{~F}-\mathrm{FDG}, \quad{ }^{18} \mathrm{~F}$-fluorodeoxyglucose; $\quad$ CCRT, concurrent chemoradiotherapy; CI, confidence interval; MTV, metabolic tumor volume; NSCLC, non-small cell lung cancer; OS, overall survival; PET/CT, positron emission tomography/computed tomography; PFS, progression-free survival; SUVmax, maximum standardized uptake value; TLG, total lesion glycolysis

Key words: preoperative concurrent chemoradiotherapy, positron emission tomography, $\Delta$ total lesion glycolysis, non-small cell lung cancer each parameter value post-CCRT. The $\mathrm{SUV}_{\max }$ threshold for defining MTV was set at 2.5. Furthermore, the association between survival and PET parameter values was analyzed. A total of 52 patients were included in the present study. The median follow-up period was 50.65 months. In univariate analysis, $\triangle \mathrm{TLG}$ was identified to be a significant predictor of progression-free survival (PFS; $\mathrm{P}=0.03$ ). The 5-year PFS rates were 48.6 and $76.6 \%$ for patients with low $\triangle$ TLG and high $\triangle T L G$, respectively. High $\triangle T L G$ was indicative of a higher overall survival rate $(\mathrm{P}=0.08)$. The present results suggest that $\triangle T L G$ calculated using PET/CT is a prognostic predictor of NSCLC treated using preoperative CCRT and surgery, and may help physicians determine treatment strategies.

\section{Introduction}

Definitive concurrent chemoradiotherapy (CCRT) is one of the standard treatments for locally advanced non-small cell lung cancer (NSCLC). Recently, a prospective trial of definitive CCRT followed by durvalumab therapy showed that prognosis was better in patients who received durvalumab than in those who received a placebo $(1,2)$. However, there did not appear to be a significant increase in the local control rates. More intensive treatments, including surgical resection, have also been attempted. Toyooka et al (3) showed that overall survival (OS) in the preoperative CCRT group was significantly better than that in the preoperative chemotherapy alone group. In a subset analysis of a phase III trial on patients with stage III NSCLC, OS in the preoperative CCRT and lobectomy group was found to be higher than that in the definitive CCRT group (4). Since not all patients benefit equally from preoperative CCRT and surgery, the 
attending physician must reasonably select the treatment options in the context of delivering personalized medicine. Moreover, research on biomarkers that can assist in selecting a treatment regimen is required.

Historically, positron emission tomography/computed tomography (PET/CT) has been utilized for the staging of NSCLC, measuring response after treatment, and predicting the prognosis after radiotherapy (5) and surgery (6). The most well-known PET/CT parameter for the quantification of ${ }^{18} \mathrm{~F}$-fluorodeoxyglucose $\left({ }^{18} \mathrm{~F}\right.$-FDG) metabolism is the maximum standardized uptake value $\left(\mathrm{SUV}_{\max }\right)$, which is the maximum voxel value in the tumor (5-7). However, $\mathrm{SUV}_{\max }$ does not indicate the overall tumor metabolic activity and is sensitive to image noise (8). Volumetric PET parameters like total lesion glycolysis (TLG) and metabolic tumor volume (MTV) have been extensively evaluated and are considered more comprehensive parameters than $\mathrm{SUV}_{\max }$ for assessing NSCLC patients (9-15). MTV was defined as the total tumor volume above threshold, while the TLG values were calculated by multiplying the target lesion mean SUV by the MTV. However, only one group has examined volumetric PET parameters as prognostic factors for preoperative CCRT and surgery $(16,17)$.

The aim of this study was to explore whether MTV and TLG calculated using PET/CT are predictors of the prognosis of NSCLC patients who have undergone preoperative CCRT and surgery.

\section{Materials and methods}

Patients. Our hospital's review board approved this study. The medical records of NSCLC patients who underwent preoperative CCRT and surgery between April 2006 and March 2018 at our hospital were retrospectively reviewed. We used the 7th edition of the TNM Classification of Malignant Tumors for staging. Patients were included if they had undergone pre- and post-CCRT PET/CT examination and preoperative concurrent chemotherapy and radiotherapy (40-60 Gy/20-30 fractions), but no other treatment before the start of CCRT. Patients with apparent accumulation of radiation pneumonitis during post-CCRT PET/CT were excluded. All procedures were performed in compliance with the ethical standards of the 1964 Declaration of Helsinki and subsequent modifications. Before commencement of preoperative CCRT, we obtained written informed consent for treatment. Opportunities to opt out of the study were provided through notices displayed in the outpatient wards and on the institution's website.

Treatment. Indications for preoperative CCRT in all cases were discussed at the respiratory conference attended by thoracic surgeons, respiratory physicians and radiation oncologists and finally decided upon by board-certified thoracic surgeons. All patients received three-dimensional conformal radiotherapy using a linear accelerator (Primus, ONCOR or Mevatron, Canon Medical Systems, Tochigi, Japan). Details of radiotherapy, including targets and margins, have been previously reported $(18,19)$. Chemotherapy regimens consisted of cisplatin/docetaxel based on a previous prospective study (20), tegafur/gimeracil/oteracil (21), and carboplatin/paclitaxel.
Surgery was usually scheduled 4-6 weeks after completion of CCRT. However, it is sometimes delayed to treat the adverse effects of CCRT and to improve the patient's general condition. Therefore, PET/CT as a preoperative examination may also be delayed.

PET/CT. PET/CT scanning was performed using a Biograph 16 PET/CT scanner (Siemens Healthcare). All patients refrained from eating and drinking for at least $6 \mathrm{~h}$ before the scan. ${ }^{18} \mathrm{~F}-\mathrm{FDG}(3.7 \mathrm{MBq} / \mathrm{kg}$ ) was administered intravenously, and PET/CT scanning was performed 90 min later. We used the syngo.via software (Siemens Healthcare) for the measurement of $\mathrm{SUV}_{\max }, \mathrm{MTV}$, and TLG. The volume of interest was manually positioned over the primary tumor and metastatic lymph node on the PET/CT image, and the contour of the target lesion (primary tumor and metastatic lymph node) inside the volume of interest was automatically delineated using the isocontour threshold method. A SUV $\mathrm{max}_{\text {max }}$ threshold of 2.5 was used to define the MTV. In a study by Im et al (8), fixed absolute thresholds were found to be suitable for the evaluation of the prognostic value of MTV; another study reported that the cutoff fixed SUV value was 2.5 in seven of the 13 studies analyzed (22). Therefore, MTV2.5 and TLG2.5 were among the volumetric PET/CT parameters measured in this study. The success of the target lesion segmentation was visually assessed by a board-certificated radiologist blinded to the patients' prognoses. SUV $\mathrm{Sax}_{\text {max }}$ MTV, and TLG values for the delineated target lesion were automatically calculated. MTV was defined as total tumor volume with ${ }^{18} \mathrm{~F}$-FDG uptake value above the threshold. TLG values were calculated by multiplying the target lesion mean SUV by the MTV. In addition, we calculated the post-CCRT percentage decrease $(\Delta)$ in each parameter value.

Statistical analysis. Early complications within 30 days of surgery were determined by a thoracic surgeon. We used the Kaplan-Meier method to obtain the survival curves. Variables were classified into two categories for the statistical analysis, and the medians were adopted as the cutoff values for the continuous PET parameters variables. The relationships between the factors and survival rates were then analyzed. The log-rank test was used for univariate analysis. A factor of two-sided $\mathrm{P}<0.05$ was determined to be statistically significant. The R software (version 3.5.1, R Foundation for Statistical Computing) was used for all analyses.

\section{Results}

Patient characteristics. Fifty-two patients were included in this study (Table I). The median follow-up period after CCRT was 50.65 (range, 6.97-139.23) months. The total preoperative radiotherapy dose was $40 \mathrm{~Gy} / 20$ fractions in two patients, $46 \mathrm{~Gy} / 23$ fractions in 47 patients, $48 \mathrm{~Gy} / 24$ fractions in one patient, and $60 \mathrm{~Gy} / 30$ fractions in two patients.

The median pre-CCRT pre-SUV max $_{\text {, }}$, pre-MTV, and pre-TLG values were 15.37 (range, 4.93-32.17), $56.84 \mathrm{~cm}^{3}$ (range, 2.64-305.16 $\mathrm{cm}^{3}$ ), and 312.03 (range, 9.53-2251.7), respectively. The median $\Delta \mathrm{SUV}_{\max }, \Delta \mathrm{MTV}$, and $\Delta \mathrm{TLG}$ were $75.72 \%$ (range, $10.70-100.00 \%$ ), $97.36 \%$ (range, $23.35-100.00 \%$ ), and $98.56 \%$ (range, 25.20-100.00\%), respectively. 
Table I. Patient characteristics.

\begin{tabular}{lcc}
\hline Characteristics & Value & Percentage \\
\hline Age (years), median & $63(35-80)$ & - \\
(range) & & \\
T stage, $\mathrm{n}$ & & 11 \\
1 & 7 & 25 \\
2 & 13 & 35 \\
3 & 18 & 27 \\
4 & 14 & \\
N stage, n & & 23 \\
0 & 12 & 10 \\
1 & 5 & 57 \\
2 & 30 & 10 \\
3 & 5 & 2 \\
Clinical stage, n & & 13 \\
II A & 1 & 58 \\
II B & 7 & 27 \\
III A & 30 & \\
III B & 14 &
\end{tabular}

Histology, $\mathrm{n}$

Adenocarcinoma

Squamous cell carcinoma

Non-small cell carcinoma

Undifferentiated carcinoma

Class V (cytology only)

Smoking history, $\mathrm{n}$

Never
Former
Current
ECOG-PS ${ }^{\mathrm{a}}, \mathrm{n}$
0
1
2
Lobe, $\mathrm{n}$
Upper
Middle
Lower
Laterality ${ }^{\mathrm{a}}, \mathrm{n}$
Right
Left

FEV1 (1) ${ }^{\mathrm{a}}$, median (range)

$\% \mathrm{VC}(\%)^{\mathrm{a}}$, median (range)

Chemotherapy, $\mathrm{n}$

Cisplatin + docetaxel

Carboplatin + paclitaxel

Tegafur/gimeracil/oteracil

Radiation dose (Gy),

median (range)

Surgery, $\mathrm{n}$

Wedge resection

Lobectomy

Bilobectomy

Pneumonectomy

$\mathrm{SUV}_{\max }$, median (range)
Table I. Continued.

\begin{tabular}{lcc}
\hline Characteristics & Value & Percentage \\
\hline $\begin{array}{l}\text { MTV2.5 }\left(\mathrm{cm}^{3}\right), \text { median } \\
\text { (range) }\end{array}$ & $56.84(2.64-305.16)$ & - \\
$\begin{array}{l}\text { TLG2.5, median (range) } \\
\Delta \text { SUV }\end{array}$ max $(\%)$, median & $75.72 .03(9.53-2251.7)$ & - \\
$($ range) & & - \\
$\begin{array}{l}\Delta \text { MTV2.5 }(\%), \text { median } \\
\text { (range) }\end{array}$ & $97.36(23.35-100.00)$ & - \\
$\begin{array}{l}\Delta \text { TLG2.5 }(\%), \text { median } \\
\text { (range) }\end{array}$ & $98.56(25.20-100.00)$ & - \\
\hline
\end{tabular}

${ }^{a}$ These factors have missing values because there were no values left in the medical records of several patients. ECOG-PS, Eastern Cooperative Oncology Group performance status; FEV1, forced expiratory volume in $1 \mathrm{sec} ; \% \mathrm{VC}$, vital capacity percentage; SUVmax, maximum standardized uptake value; MTV2.5, metabolic tumor volume of $\mathrm{SUV}_{\max }>2.5$; TLG2.5, total lesion glycolysis of SUVmax $>2.5$.

PET parameters and prognosis. Table II shows the factors associated with PFS. In univariate analysis, T-stage, forced expiratory volume in $1 \mathrm{sec}$, and $\triangle \mathrm{TLG}$ were found to be significant predictors of PFS $(\mathrm{P}=0.02,0.04$, and 0.03 , respectively). Pre-SUV $V_{\max }, \mathrm{MTV}, \mathrm{TLG}, \Delta \mathrm{SUV}_{\max }$, and $\triangle \mathrm{MTV}$ were not predictors of PFS $(\mathrm{P}=0.4,0.2,0.1,0.09$, and 0.09 , respectively). Fig. 1 shows the PFS Kaplan-Meier curve for the high and low $\triangle T L G$ groups. In the low $\triangle \mathrm{TLG}$ group, the 3, 5 and 10-year PFS rates were $48.6 \%$ [95\% confidence interval (CI), 32.9-71.8\%], 48.6\% (95\% CI, 32.9-71.8\%), and not-reached, respectively. In the high $\Delta \mathrm{TLG}$ group, the corresponding rates were $87.5 \%$ (95\% CI, 75.2-100\%), 76.6\% (95\% CI, 66.3-97.2\%), and $56.7 \%$ (95\% CI, 34.8-92.4\%), respectively.

Table III shows the factors associated with OS. In univariate analysis, $\mathrm{SUV}_{\max }, \mathrm{MTV}, \mathrm{TLG}, \Delta \mathrm{SUV}_{\max }$, and $\triangle \mathrm{MTV}$ were not found to be associated with OS. High $\triangle T L G$ was indicative of a higher OS rate $(\mathrm{P}=0.08)$. In the low $\triangle \mathrm{TLG}$ group, the 3,5 , and 10-year OS rates were $76.5 \%$ (95\% CI, 61.6-95.0\%), $58.3 \%$ (95\% CI, 39.9-85.1\%), and 58.3\% (95\% CI, 39.9-85.1\%), respectively. In the high $\triangle \mathrm{TLG}$ group, the corresponding rates were $100 \%$ (not applicable), 88.4\% (95\% CI, 74.5-100\%), and 50.2\% (95\% CI, 24.6-100\%), respectively (Fig. 2). Pneumonectomy was found to be associated with a low OS rate $(\mathrm{P}=0.03)$.

Other prognostic factors and complications. The median time from the last day of CCRT to post-CCRT PET/CT was 3.57 weeks (range, 1.71-9.00 weeks). The 5-year survival rates for the shorter time group below the median and longer time group above the median were $68.8 \%$ (95\% CI, 50.5-93.7\%) and $76.3 \%$ (95\% CI, 59.7-97.6\%), respectively, which were not significantly different $(\mathrm{P}=0.5)$. The median time from the last day of CCRT to surgery was 5.71 weeks (range, 3.14-12.86 weeks). The 5-year survival rates for shorter time group below the median and longer time group above the median were $66.7 \%$ (95\% CI, 48.9-91.1\%) and $80.1 \%$ (95\% CI, $64.2-100 \%)$, respectively, which were not significantly different 
Table II. Univariate analysis of factors associated with progression-free survival.

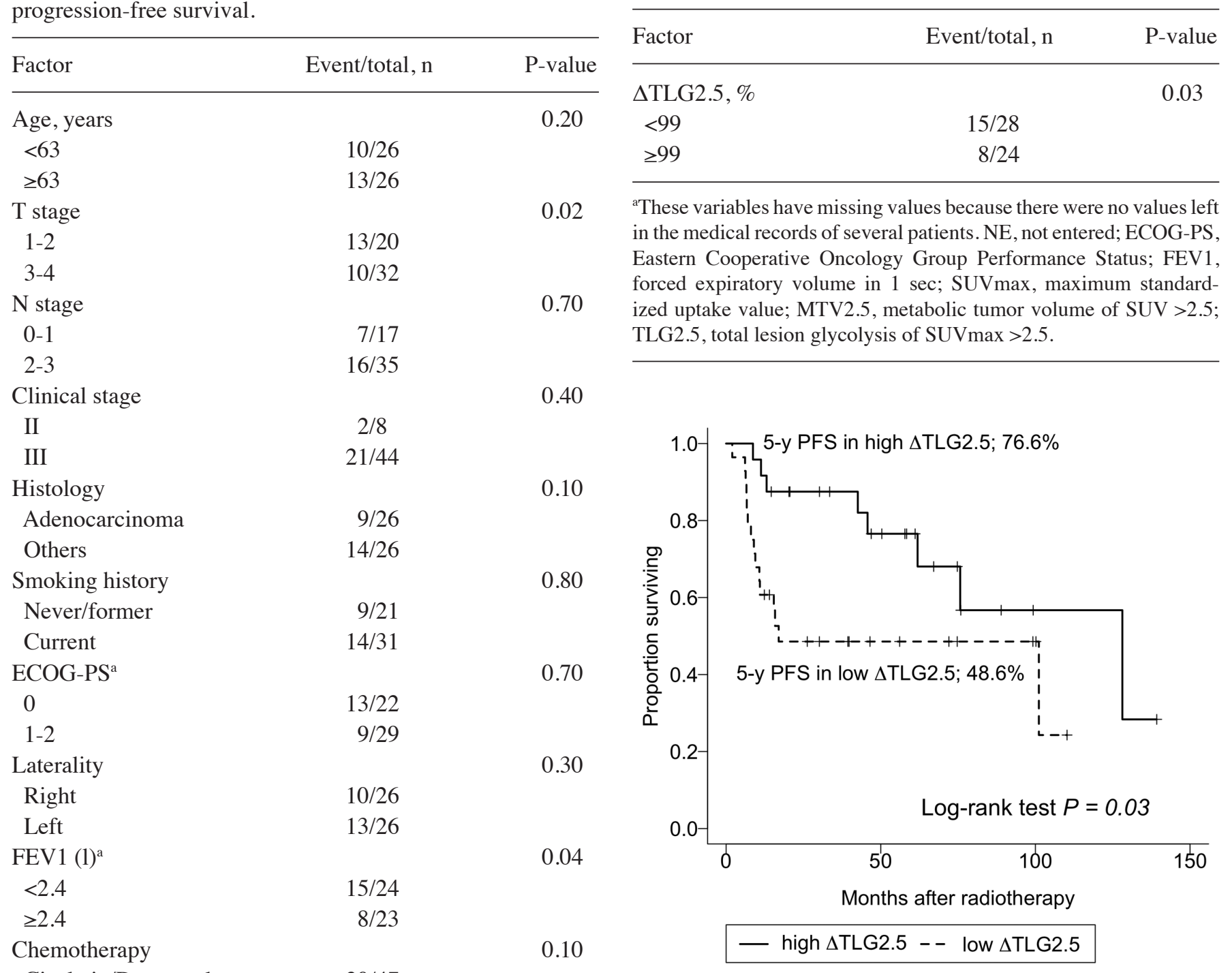

Cisplatin/Docetaxel 20/47

Others $\quad 3 / 5$

Radiation dose, Gy

$<60$

$22 / 50$

60

$1 / 2$

Surgery

Pneumonectomy

Others

$\mathrm{SUV}_{\text {max }}$

$<15$

$13 / 26$

$\geq 15$

$10 / 26$

MTV2.5, $\mathrm{cm}^{3}$

$<57$

$14 / 25$

$\geq 57$

$9 / 27$

TLG2.5

$<310$

$12 / 26$

$\geq 310$

$9 / 26$

$\Delta \mathrm{SUV}_{\text {max }}, \%$

$<76$

$13 / 26$

$\geq 76$

$10 / 26$

$\triangle \mathrm{MTV} 2.5, \%$

$<97$

$13 / 25$

$\geq 97$
Table II. Continued.

aThese variables have missing values because there were no values left in the medical records of several patients. NE, not entered; ECOG-PS, Eastern Cooperative Oncology Group Performance Status; FEV1, orced expiratory volume in $1 \mathrm{sec}$; SUVmax, maximum standardTLG2.5, total lesion glycolysis of SUVmax $>2.5$.

Figure 1. Subgroup analysis of progression-free survival. Proportion of patients surviving shown by number of months after radiotherapy, with the high $\triangle \mathrm{TLG}$ group presented as the filled line and the low $\triangle \mathrm{TLG}$ group glycolysis; 5-y PFS, 5-year progression-free survival.

( $\mathrm{P}=0.3$ ) (Fig. 3). There were 33 patients with early postoperative complications within 30 days of surgery; the 5-year survival rates for the groups with and without early complications were $69.9 \%$ (95\% CI, 54.1-90.3\%) and 78.1\% (95\% CI, 58.3-100\%), respectively, which were not significantly different $(\mathrm{P}=0.8)$.

0.20 The 33 patients had the following complications: Arrhythmia, 6 patients; radiation pneumonitis, 4 patients; recurrent nerve palsy, 3 patients; bacterial pneumonia, 3 patients; pleural effusion, 2 patients; chylothorax, 2 patients; pyothorax plus enteritis, prolonged pulmonary fistula, functional pyloric ring stenosis, chylothorax plus bacterial pneumonia plus arrhythmia, arrhythmia plus sepsis from pneumonia, atelectasis, chylothorax plus pulmonary artery embolus plus venous thrombosis, wound infection, recurrent laryngeal nerve palsy plus arrhythmia plus venous thrombosis, upper limb paresis plus recurrent laryngeal nerve paralysis, radiation pneumonitis plus recurrent laryngeal nerve palsy, venous thrombosis, and upper gastrointestinal bleeding, 1 patient. Postoperative death presented as the dotted line. $\Delta$, percentage decrease; TLG, total lesion 
Table III. Univariate analysis of factors associated with overall survival.

\begin{tabular}{|c|c|c|}
\hline Factor & Event/total, $\mathrm{n}$ & P-value \\
\hline
\end{tabular}

\begin{tabular}{lr}
\hline Age, years & \\
$<63$ & $8 / 26$ \\
$\geq 63$ & $8 / 26$ \\
T stage & \\
$1-2$ & $10 / 20$ \\
$3-4$ & $6 / 32$ \\
N stage &
\end{tabular}

$0-1 \quad 5 / 17$

2-3 11/35

Clinical stage

II

III

Histology

Adenocarcinoma

Others

Smoking history

Never/former

Current

ECOG-PS ${ }^{\mathrm{a}}$

0

1-2

Laterality

Right

Left

FEV1 (1) ${ }^{\mathrm{a}}$

$<2.4$

$\geq 2.4$

Chemotherapy

Cisplatin/docetaxel

Others

Radiation dose, Gy

$<60$

60

Surgery

Pneumonectomy

Others

$\mathrm{SUV}_{\text {max }}$

$<15$

MTV2.5, $\mathrm{cm}^{3}$

$<57$

$\geq 57$

TLG2.5

$<310$

$\geq 310$

$\triangle \mathrm{SUV}_{\text {max }} \%$

$<76$

$\geq 76$

$\triangle \mathrm{MTV} 2.5, \%$

$<97$

$\geq 97$
$1 / 8$

$15 / 44$

$9 / 26$

$7 / 26$

$6 / 21$

$10 / 31$

$10 / 29$

$5 / 22$

$7 / 26$

$9 / 26$

$10 / 24$

$6 / 23$

$14 / 47$

$2 / 5$

$15 / 50$

$1 / 2$

$2 / 3$

$14 / 49$

$8 / 26$

$8 / 26$

$9 / 25$

$7 / 27$

$9 / 26$

$7 / 26$

0.30

0.06

0.90

0.70

0.40

0.70

0.50

0.20

0.08

0.50

0.03

0.90

0.50

$9 / 26$

$7 / 26$

$11 / 25$

$6 / 27$
Table III. Continued.

\begin{tabular}{lcr}
\hline Factor & Event/total, & P-value \\
\hline$\Delta$ TLG2.5, & & 0.08 \\
$<99$ & $10 / 28$ & \\
$\geq 99$ & $6 / 24$ &
\end{tabular}

${ }^{a}$ These variables have missing values because there were no values left in the medical records of several patients. NE, not entered; ECOG-PS, Eastern Cooperative Oncology Group Performance Status; FEV1, forced expiratory volume in $1 \mathrm{sec}$; SUVmax, maximum standardized uptake value; MTV2.5, metabolic tumor volume of SUV >2.5; TLG2.5, total lesion glycolysis of SUVmax $>2.5$.

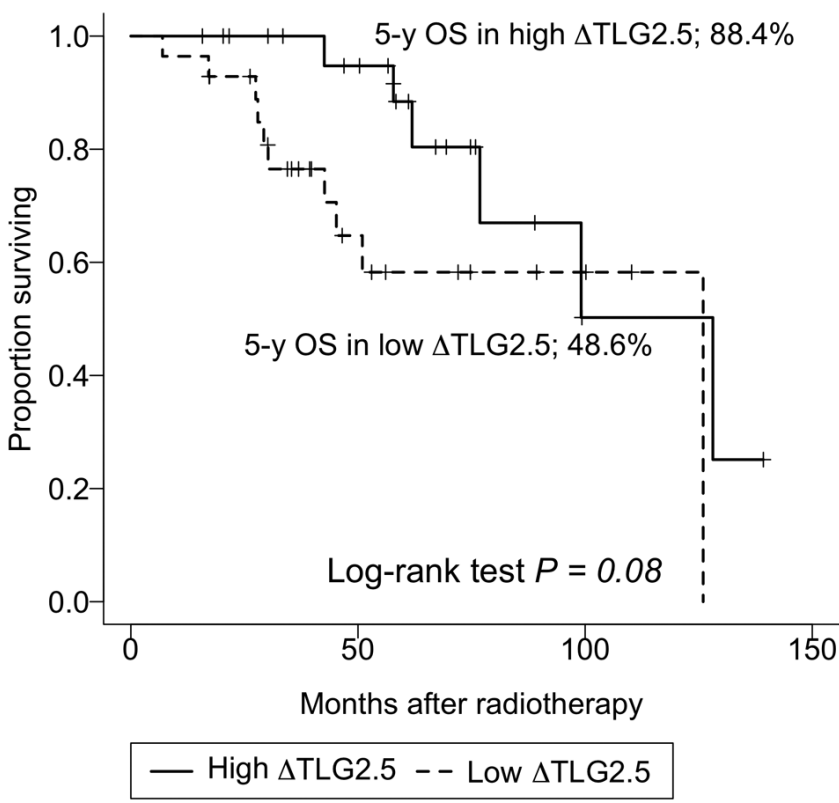

Figure 2. Subgroup analysis of overall survival. Proportion of patients surviving shown by number of months after radiotherapy, with the high $\Delta T L G$ group presented as the filled line and the low $\triangle \mathrm{TLG}$ group presented as the dotted line. $\Delta$, percentage decrease; TLG, total lesion glycolysis; $5-y$ OS, 5-year overall survival.

due to complications was not observed in any of the 33 patients here assessed.

Clinical course. Of the 52 patients included, there were 29 survivors without recurrence, seven survivors with recurrence, 11 deaths due to NSCLC, and five deaths due to conditions other than NSCLC. In the low $\triangle \mathrm{TLG}$ group, there were 13 survivors without recurrence, five survivors with recurrence, eight deaths due to NSCLC, and two deaths due to conditions other than NSCLC. In the high $\triangle T L G$ group, there were 16 survivors without recurrence, two survivors with recurrence, three deaths due to NSCLC, and three deaths due to conditions other than NSCLC.

\section{Discussion}

In our study, $\triangle \mathrm{TLG}$ was found to be a prognostic factor for PFS. Previous investigators have reported that $\mathrm{SUV}_{\max }$ is 


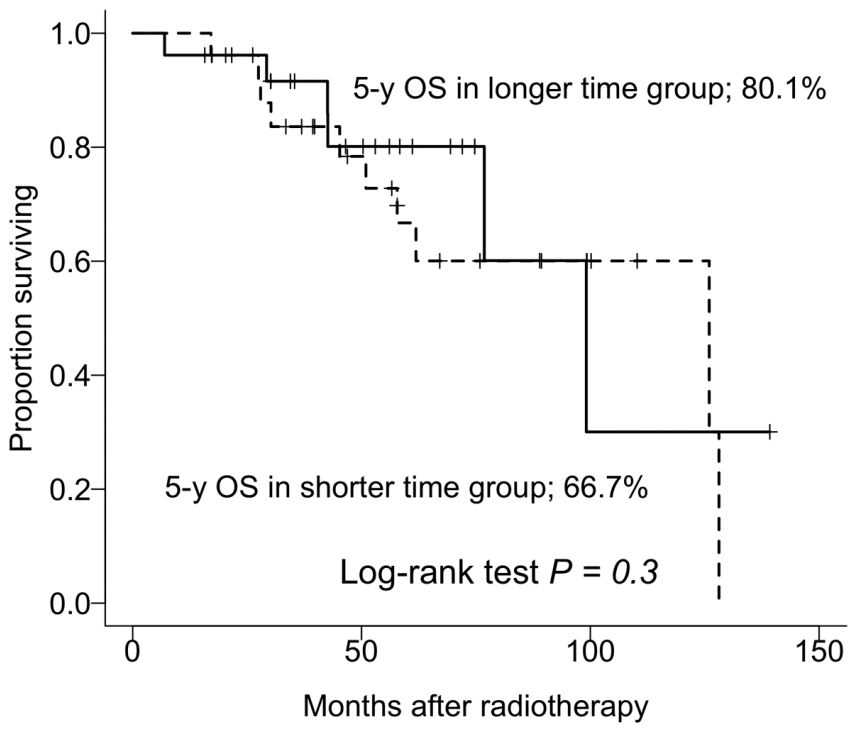

-Longer time group - - Shorter time group

Figure 3. Subgroup analysis of overall survival stratified according to time from the last day of concurrent chemoradiotherapy to surgery. Proportion of surviving patients shown by number of months after radiotherapy, where the filled line indicates the longer time group (above the median), while the dotted line indicates the shorter time group (below the median). 5-y OS, 5-year overall survival.

a predictor of PFS and OS for NSCLC patients receiving surgery. Liu et al (6) performed a meta-analysis that evaluated the correlation between PET parameters and prognosis of stage I-IV NSCLC patients who had undergone surgery. In this study, most of the evaluated PET parameters were $\mathrm{SUV}_{\max }$ values. In a systematic review by Nair et al (7), high FDG uptake was found to be related to poor prognosis in patients who underwent surgical treatment for stage I NSCLC; $\mathrm{SUV}_{\max }$ was measured in six of the nine articles included in the analysis. Another meta-analysis examining the significance of pretreatment SUV $\mathrm{Sax}_{\text {max }}$ in NCLC patients who had undergone definitive radiotherapy showed that pre-radiotherapy tumor $\mathrm{SUV}_{\max }$ can predict outcomes (5). The combined hazard ratio of pre-radiotherapy $\mathrm{SUV}_{\max }$ was 1.05 (95\% CI, 1.02-1.08) for OS and 1.26 (95\% CI, 1.05-1.52) for local control. However, other investigators have shown that volumetric PET parameters are more predictive of patient survival than $\mathrm{SUV}_{\max }$ in surgical cases of early stage NSCLC $(9,15)$. Recent reports on early stage (stages I-II) NSCLC have also documented the utility of MTV and TLG (10-11). In a study conducted by Shrestha et al (10), MTV was found to be a predictive factor of PFS in stage I NSCLC patients treated with carbon ion particle therapy. A study of PET parameters and prognosis in stereotactic radiotherapy for early stage NSCLC patients showed that only volumetric parameters were significant predictors of PFS for patients with large primary tumors (11). Volumetric parameters have also been examined in locally advanced cases. Grootjans et al (12) demonstrated that, for 27 stage III NSCLC patients who underwent definitive chemoradiotherapy, pretreatment TLG was a significant predictor of PFS and OS. In a prospective study, pretreatment $\mathrm{SUV}_{\max }$ was not found to be a predictor for locally advanced NSCLC treated using definitive CCRT (13). Using the same clinical trial dataset, Salavati et al (14) showed that the areas under the curves for volumetric parameters were higher than those for $\mathrm{SUV}_{\max }$, and the prognoses of the high MTV and TLG groups were significantly worse than those of the low MTV and TLG groups. Hyun et al (16) demonstrated that MTV and TLG were significant predictive factors of prognosis in a multivariate analysis of operative stage III cases. At later stages, volumetric PET parameters are considered more important than $\mathrm{SUV}_{\max }$ as prognostic factors. Our results also show that TLG calculated using PET/CT was a useful predictor of prognosis and are consistent with these previous reports.

In our study, neither $\mathrm{SUV}_{\max }$ nor $\Delta S U \mathrm{~V}_{\max }$ was a significant predictor of PFS or OS. The FDG metabolic imaging method evaluates tumor biology by measuring tumor SUVs, and the major method of SUV measurement based on $\mathrm{SUV}_{\max }$. Since this only reflects a single voxel value indicating the highest uptake of FDG in the tumor, $\mathrm{SUV}_{\max }$ does not necessarily represent the overall burden or metabolic activity of a tumor $(8,23)$. Further, SUV is influenced by various factors, including length of the uptake period, body composition, recovery coefficient, plasma glucose level, image noise, and partial volume effects (24,25). As MTV and TLG consider not only single voxel information but also highly active three-dimensional tumor volume and metabolic activity, clinicians can use them to obtain more detailed tumor information.

Pre-CCRT PET parameters were not found to be prognostic factors in our study. In a study on 161 patients who underwent preoperative CCRT, Hyun et al (17) showed that pre-CCRT MTV was a predictor of prognosis. The chemotherapy regimen in their study consisted of paclitaxel or docetaxel plus either cisplatin or carboplatin, although the proportions were not specified. In our study, cisplatin/docetaxel was used for most patients, and the differences between our results and theirs may be due to the differences in the size of the patient cohort, institutional treatment strategies, and technical factors.

The correlations between the percentage decrease in volumetric PET parameter values and prognosis of locally advanced NSCLC treated using definitive CCRT has been investigated previously. In the study by Huang et al (23), only $\triangle \mathrm{MTV}$ was found to be a predictor of OS according to a Cox regression analysis of NSCLC patients treated with definitive CCRT. Grootjans et al (12) demonstrated that $\triangle \mathrm{TLG}$ was significantly associated with prognosis. To the best of our knowledge, no studies have examined the significance of the percentage decrease in volumetric PET parameter values after preoperative CCRT and surgery, and further studies in this context are warranted. In our study, high $\triangle \mathrm{TLG}$ was indicative of a high OS rate, but the correlation was not significant $(\mathrm{P}=0.08)$. There were five living patients with recurrence in the low $\triangle \mathrm{TLG}$ groups and two in the high $\triangle T L G$ group. In addition to the small number of cases, the difference in the number of living patients with recurrence is likely to be one of the reasons why, despite the difference in PFS, there was no difference in OS between the low and high $\triangle T L G$ groups. The greatest advantage of utilizing this parameter as a prognostic factor is that, in addition to measuring the size of the tumor (as done by CT), measuring the rate of decrease in activity of the entire tumor volume will be possible. However, limitations exist, including i) the 
unavailability of a decrease rate index due to the lack of evidence other than ours in patients undergoing preoperative CCRT; ii) the additional costs associated with PET imaging; and iii) the exposure to radiation, which does not concern CT instead.

Volumetric PET parameters may accurately reflect prognosis in NSCLC patients undergoing preoperative CCRT and surgery. The prognosis of patients receiving definitive CCRT is significantly improved by the addition of durvalumab after definitive CCRT $(1,2)$. In the future, volumetric evaluation using PET/CT in the clinical setting may enable personalized treatment strategies tailored to each patient's prognostic risk. Patients who are expected to have a high likelihood of recurrence after preoperative CCRT, with additional evidence based on predictive biomarkers such as $\triangle \mathrm{TLG}$, may be recommended to receive definitive CCRT and an immune checkpoint inhibitor. With regard to patient follow-up after treatment, a grasp of patient clinical information using online resources is known to improve the prognosis of patients with lung cancer (26). Another potential significance of volumetric PET parameter evaluation could be that rigorous post-treatment follow-up of patients with low $\triangle T L G$ could lead to the rapid induction of treatment after NSCLC recurrence.

Nonetheless, our study has some limitations. First, this study was retrospective in nature and involved a single center; therefore, there may have been some undetectable biases. Second, the PET/CT scans were performed 90 min after injection of FDG, while it is generally recommended that imaging be performed $60 \mathrm{~min}$ after injection (27). Third, this study included only a small number of patients. Further multi-institutional and prospective studies are necessary to confirm the prognostic value of $\triangle T L G$.

In conclusion, we demonstrated that $\triangle \mathrm{TLG}$ was a predictor of prognosis in NSCLC patients treated using preoperative CCRT and surgery. Our results suggest that $\triangle$ TLG calculated using PET/CT could help physicians determine treatment strategies for locally advanced NSCLC.

\section{Acknowledgements}

The authors would like to thank Dr Shimpei Tsudaka and Dr Hiromasa Yamamoto (Department of Thoracic Surgery, Okayama University Hospital, Okayama, Japan) for data collection.

\section{Funding}

The present study was supported by a donation from Tsuyama Chuo Hospital. The study sponsor was not involved in any procedure in the present study.

\section{Availability of data and materials}

All data generated or analyzed during this study are included in this published article.

\section{Authors' contributions}

KKa contributed to the design of the study, collected the data and drafted the manuscript. TO contributed to the design of the study and performed statistical analysis. AT and KW contributed to the design of the study and collected the data. The raw data have been assessed by KKa and TO. KY, MK, $\mathrm{KKi}, \mathrm{TH}, \mathrm{ST}$ and SK contributed to the design of the study. All authors read and approved the final manuscript.

\section{Ethics approval and consent to participate}

The Okayama University Graduate School of Medicine, Dentistry and Pharmaceutical Sciences and Okayama University Hospital, Ethics Committee (Okayama, Japan) approved the present study (approval no. 1809-018). Written informed consent was obtained prior to treatment. The choice to opt-out was provided through notifications displayed on the hospital's website and in the outpatient ward before the start of the present study.

\section{Patient consent for publication}

Not applicable.

\section{Competing interests}

The authors declare that they have no competing interests.

\section{References}

1. Antonia SJ, Villegas A, Daniel D, Vicente D, Murakami S, Hui R, Yokoi T, Chiappori A, Lee KH, de Wit M, et al: Durvalumab after chemoradiotherapy in stage III non-small-cell lung cancer. N Engl J Med 377: 1919-1929, 2017.

2. Antonia SJ, Villegas A, Daniel D, Vicente D, Murakami S, Hui R, Kurata T, Chiappori A, Lee KH, de Wit M, et al: Overall survival with durvalumab after chemoradiotherapy in stage III NSCLC. N Engl J Med 379: 2342-2350, 2018

3. Toyooka S, Kiura K, Takemoto M, Oto T, Takigawa N, Fujiwara T, Miyoshi S and Date H: Long-term outcome of induction chemoradiotherapy with docetaxel and cisplatin followed by surgery for non-small-cell lung cancer with mediastinal lymph node metastasis. Interact Cardiovasc Thorac Surg 14: 565-569, 2012.

4. Albain KS, Swann RS, Rusch VW, Turrisi AT III, Shepherd FA, Smith C, Chen Y, Livingston RB, Feins RH, Gandara DR, et al: Radiotherapy plus chemotherapy with or without surgical resection for stage III non-small-cell lung cancer: A phase III randomised controlled trial. Lancet 374: 379-386, 2009.

5. Na F, Wang J, Li C, Deng L, Xue J and Lu Y: Primary tumor standardized uptake value measured on F18-Fluorodeoxyglucose positron emission tomography is of prediction value for survival and local control in non-small-cell lung cancer receiving radiotherapy: Meta-analysis. J Thorac Oncol 9: 834-842, 2014.

6. Liu J, Dong M, Sun X, Li W, Xing L and Yu J: Prognostic value of ${ }^{18} \mathrm{~F}-\mathrm{FDG}$ PET/CT in surgical non-small cell lung cancer: A meta-analysis. PLoS One 11: e0146195, 2016.

7. Nair VS, Krupitskaya Y and Gould MK: Positron emission tomography ${ }^{18} \mathrm{~F}$-fluorodeoxyglucose uptake and prognosis in patients with surgically treated, stage I non-small cell lung cancer: A systematic review. J Thorac Oncol 4: 1473-1479, 2009.

8. Im HJ, Bradshaw T, Solaiyappan M and Cho SY: Current methods to define metabolic tumor volume in positron emission tomography: Which one is better? Nucl Med Mol Imaging 52: $5-15,2018$.

9. Park SY, Cho A, Yu WS, Lee CY, Lee JG, Kim DJ and Chung KY: Prognostic value of total lesion glycolysis by ${ }^{18} \mathrm{~F}-\mathrm{FDG}$ PET/CT in surgically resected stage IA non-small cell lung cancer. J Nucl Med 56: 45-49, 2015.

10. Shrestha S, Higuchi T, Shirai K, Tokue A, Shrestha S, Saitoh JI, Hirasawa H, Ohno T, Nakano T and Tsushima Y: Prognostic significance of semi-quantitative FDG-PET parameters in stage I non-small cell lung cancer treated with carbon-ion radiotherapy. Eur J Nucl Med Mol Imaging 47: 1220-1227, 2019. 
11. Satoh Y, Onishi H, Nambu A and Araki T: Volume-based parameters measured by using FDG PET/CT in patients with stage I NSCLC treated with stereotactic body radiation therapy: Prognostic value. Radiology 270: 275-281, 2014.

12. Grootjans W, Usmanij EA, Oyen WJ, van der Heijden EH Visser EP, Visvikis D, Hatt M, Bussink J and de Geus-Oei LF: Performance of automatic image segmentation algorithms for calculating total lesion glycolysis for early response monitoring in non-small cell lung cancer patients during concomitant chemoradiotherapy. Radiother Oncol 119: 473-479, 2016.

13. Machtay M, Duan F, Siegel BA, Snyder BS, Gorelick JJ, Reddin JS, Munden R, Johnson DW, Wilf LH, DeNittis A, et al: Prediction of survival by $\left[{ }^{18} \mathrm{~F}\right]$ fluorodeoxyglucose positron emission tomography in patients with locally advanced non-small-cell lung cancer undergoing definitive chemoradiation therapy: Results of the ACRIN 6668/RTOG 0235 trial. J Clin Oncol 20: 3823-3830, 2013.

14. Salavati A, Duan F, Snyder BS, Wei B, Houshmand S, Khiewvan B, Opanowski A, Simone CB II, Siegel BA, Machtay M and Alavi A: Optimal FDG PET/CT volumetric parameters for risk stratification in patients with locally advanced non-small cell lung cancer: Results from the ACRIN 6668/RTOG 0235 trial. Eur J Nucl Med Mol Imaging 44: 1969-1983, 2017.

15. Hyun SH, Choi JY, Kim K, Kim J, Shim YM, Um SW, Kim H, Lee KH and Kim BT: Volume-based parameters of (18) F-fluorodeoxyglucose positron emission tomography/computed tomography improve outcome prediction in early-stage non-small cell lung cancer after surgical resection. Ann Surg 257: 364-370, 2013.

16. Hyun SH, Ahn HK, Kim H, Ahn MJ, Park K, Ahn YC, Kim J, Shim YM and Choi JY: Volume-based assessment by (18)F-FDG $\mathrm{PET} / \mathrm{CT}$ predicts survival in patients with stage III non-small-cell lung cancer. Eur J Nucl Med Mol Imaging 41: 50-58, 2014.

17. Hyun SH, Ahn HK, Ahn MJ, Ahn YC, Kim J,Shim YM and Choi JY: Volume-based assessment with ${ }^{18} \mathrm{~F}-\mathrm{FDG}$ PET/CT improves outcome prediction for patients with stage IIIa-N2 non-small cell lung cancer. AJR Am J Roentgenol 205: 623-628, 2015.

18. Ogata T, Katsui K, Yoshio K, Ihara H, Katayama N, Soh J, Kuroda M, Kiura K, Maeda Y, Toyooka S and Kanazawa S: Dose-volume parameters predict radiation pneumonitis after surgery with induction concurrent chemoradiotherapy for non-small cell lung cancer. Acta Med Okayama 72: 507-513, 2018.

19. Katsui K, Ogata T, Watanabe K, Katayama N, Kuroda M, Kiura K, Hiraki T, Maeda Y, Toyooka S and Kanazawa S: Radiation pneumonitis after definitive concurrent chemoradiotherapy with cisplatin/docetaxel for non-small cell lung cancer: Analysis of dose-volume parameters. Cancer Med 9: 4540-4549, 2020 .
20. Segawa Y, Kiura K, Takigawa N, Kamei H, Harita S, Hiraki S, Watanabe Y, Sugimoto K, Shibayama T, Yonei T, et al: Phase III trial comparing docetaxel and cisplatin combination chemotherapy with mitomycin, vindesine, and cisplatin combination chemotherapy with concurrent thoracic radiotherapy in locally advanced non-small-cell lung cancer: OLCSG 0007. J Clin Oncol 28: 3299-3306, 2010.

21. Takigawa N, Kiura K, Hotta K, Hosokawa S, Nogami N, Aoe K, Gemba K, Fujiwara K, Harita S, Takemoto M, et al: A phase I study of S-1 with concurrent thoracic radiotherapy in elderly patients with localized advanced non-small cell lung cancer. Lung Cancer 71: 60-64, 2011.

22. Im HJ, Pak K, Cheon GJ, Kang KW, Kim SJ, Kim IJ, Chung JK, Kim EE and Lee DS: Prognostic value of volumetric parameters of (18)F-FDG PET in non-small-cell lung cancer: A meta-analysis. Eur J Nucl Med Mol Imaging 42: 241-251, 2015.

23. Huang W, Fan M, Liu B, Fu Z, Zhou T, Zhang Z, Gong H and $\mathrm{Li} \mathrm{B}$ : Value of metabolic tumor volume on repeated ${ }^{18} \mathrm{~F}-\mathrm{FDG}$ PET/CT for early prediction of survival in locally advanced non-small cell lung cancer treated with concurrent chemoradiotherapy. J Nucl Med 55: 1584-1590, 2014.

24. Keyes JW Jr: SUV: Standard uptake or silly useless value? J Nucl Med 36: 1836-1839, 1995

25. Boellaard R, Krak NC, Hoekstra OS and Lammertsma AA: Effects of noise, image resolution, and ROI definition on the accuracy of standard uptake values: A simulation study. J Nucl Med 45: 1519-1527, 2004.

26. Denis F, Lethrosne C, Pourel N, Molinier O, Pointreau Y, Domont J, Bourgeois $\mathrm{H}$, Senellart $\mathrm{H}$, Trémolières $\mathrm{P}$, Lizée T, et al: Randomized trial comparing a web-mediated follow-up with routine surveillance in lung cancer patients. J Natl Cancer Inst 109, 2017.

27. Wahl RL, Jacene H, Kasamon Y and Lodge MA: From RECIST to PERCIST: Evolving considerations for PET response criteria in solid tumors. J Nucl Med 50 (Suppl 1): 122S-150S, 2009.

This work is licensed under a Creative Commons Attribution-NonCommercial-NoDerivatives 4.0 International (CC BY-NC-ND 4.0) License. 